\title{
Cardiovascular safety of methylphenidate among children and young people with attention-deficit/hyperactivity disorder (ADHD): nationwide self controlled case series study
}

\author{
Ju-Young Shin, ${ }^{1,2}$ Elizabeth E Roughead, ${ }^{3}$ Byung-Joo Park,, ${ }^{4}$ Nicole L Pratt ${ }^{3}$
}

${ }^{1}$ Center for Clinical

Epidemiology, Lady Davis

Research Institute, Jewish

General Hospital, Montreal, QC,

Canada

${ }^{2}$ Departments of Epidemiology, Biostatistics, and Occupational Health, McGill University,

Montreal, QC, Canada

${ }^{3}$ School of Pharmacy and

Medical Sciences, University of

South Australia, Adelaide SA

5001, Australia

${ }^{4}$ Department of Preventive

Medicine, Seoul National

University College of Medicine, Seoul, Korea

Correspondence to: N L Pratt Nicole.Pratt@unisa.edu.au

Additional material is published online only. To view please visit

Cite this as: BMJ 2016;353:i2550 http://dx.doi.org/10.1136/bmj.i2550

Accepted: 25 April 2016 the journal online.

\author{
ABSTRACT \\ OBJECTIVE \\ To determine whether treatment with methylphenidate \\ in children and young people with attention-deficit/ \\ hyperactivity disorder (ADHD) was associated with \\ cardiovascular events.
}

DESIGN

Self controlled case series analysis.

SETTING

Nationwide health insurance database, 1 January 2008 to 31 December 2011, in South Korea.

\section{PARTICIPANTS}

1224 patients aged $\leq 17$ who had experienced an incident cardiovascular event and had had at least one incident prescription for methylphenidate.

\section{MAIN OUTCOME MEASURES}

A recorded diagnosis (either a primary or secondary cause) of any of the following cardiovascular adverse events: arrhythmias (ICD-10 (international classification of diseases, 10th revision) codes 144, 145, 147, 148, 149), hypertension (codes 110-115), myocardial infarction (code 121), ischemic stroke (code 163), or heart failure (code 150). Incidence rate ratios were calculated with conditional Poisson regression and adjusted for time varying comorbidity and comedication.

\section{RESULTS}

Increased risk of arrhythmia was observed in all exposed time periods-that is, periods of treatment with methylphenidate-(incidence rate ratio 1.61, 95\% confidence interval 1.48 to 1.74 ), and the risk was highest in the children who had congenital heart disease. No significant risk of myocardial infarction was observed for all exposed time periods $(1.33,0.90$ to 1.98), though risk was higher in the early risk periods between eight and 56 days after the start of treatment with methylphenidate. No significant increased risk was observed for hypertension, ischemic stroke, or heart failure.

\section{WHAT IS ALREADY KNOWN ON THIS TOPIC}

Previous observational studies have reported no association between methylphenidate use in children and young people and adverse cardiovascular events

Statistical power was limited in those studies as the absolute risk of cardiovascular events is low in children and the study designs meant that confounding could not be ruled out

\section{WHAT THIS STUDY ADDS}

Use of methylphenidate in children/young people with diagnosis of ADHD was associated with an increased risk of arrhythmia and myocardial infarction While there was an increased relative risk, the absolute risk is likely to be low No increased risk was observed for hypertension, ischemic stroke, or heart failure

\section{CONCLUSION}

The relative risk of myocardial infarction and arrhythmias is increased in the early period after the start of methylphenidate treatment for ADHD in children and young people. Though the absolute risk is likely to be low, the risk-benefit balance of methylphenidate should be carefully considered, particularly in children with mild ADHD.

\section{Introduction}

Drugs to treat attention-deficit/hyperactivity disorder (ADHD) have been shown to be efficacious in reducing symptoms of impulsivity and hyperactivity in children, ${ }^{1}$ but concerns have been expressed about possible adverse cardiovascular events with the first line treatment, methylphenidate. ${ }^{2}$ In 2009, one case-control study reported a strong association between use of stimulants in young people and sudden death $(1.8 \%, 10$ users among 564 cases) compared with use of stimulants in death in people in motor vehicle crashes $(0.4 \%$, two users among 564 controls) (odds ratio 7.4, 95\% confidence interval 1.4 to 74.9). ${ }^{3}$ Comparability between the cases and controls, infrequent exposure, and potential recall bias were limitations in this analysis.

Subsequently, large retrospective population based cohort studies have found no evidence of an increased risk of myocardial infarction or stroke associated with methylphenidate. ${ }^{4-7}$ A systematic review reported that findings of observational studies of children and adolescents were variable and suggested that statistical power was a limitation as the absolute risk of cardiovascular events was low in the populations studied and the study designs meant that confounding could not be ruled out. ${ }^{8}$ Given this uncertainty around the risks associated with methylphenidate, case reports of adverse cardiovascular events continue to be published. ${ }^{9}$ There have been five case reports of life threatening heart failure associated with methylphenidate reported from Sweden. ${ }^{10}$ Additionally, small but significant increases in blood pressure have been reported in children and adolescents. ${ }^{11}$ Despite these latter reports, no observational studies have been performed to examine the risk of heart failure or hypertensive disease in children and adolescents exposed to methylphenidate.

We explored whether methylphenidate use is a triggering factor among children who experience cardiovascular events-including arrhythmia, hypertension, myocardial infarction, ischemic stroke, or heart failure-using a self controlled case series study design. Using this technique, which is a within person design, we looked at the incidence of each cardiovascular adverse event in children during periods in which they 
were exposed to methylphenidate compared with periods in which they were not exposed.

\section{Methods}

Database

We used the South Korea national health insurance claims database. The national health insurance program was initiated in Korea in 1977 and achieved universal coverage of the entire population by 1989 . The database contains all information on the diagnoses and prescribed drugs for about 50 million Koreans. ${ }^{12} \mathrm{We}$ obtained the claims data for children and young people aged $\leq 17$ with diagnosis of ADHD (ICD-10 (international classification of diseases, 10th revision) code F90) that had been submitted by healthcare providers from 1 January 2007 to 31 December 2011. The claims database included an anonymized identifier representing each individual together with age, sex, diagnoses, and prescription drugs. Information on prescribed drugs included generic name, prescription date, duration, and route of administration.

All diagnoses are coded according to ICD-10. Previous validation studies have compared the diagnoses derived from the claims database with the ideal of actual diagnoses recorded in the patients' medical records obtained from hospital or clinic chart review. The studies reported that the positive predictive value of the diagnoses was $83.4 \%$ for ischemic stroke (code I63) and $>70 \%$ for myocardial infarction (code I21). ${ }^{13-15}$ The overall positive predictive value of all diagnoses was about $70 \% .^{13}$

\section{Self controlled case series design}

We used a self controlled case series design in this analysis. This design is derived from the cohort design and determined the incidence of cardiovascular adverse events during periods in which the participants were exposed to methylphenidate compared with the incidence in periods in which they were not exposed. We used a bi-directional study design and collected data on cardiovascular adverse events in unexposed time either before or after exposure. The study design minimizes confounding because the patients each act as their own

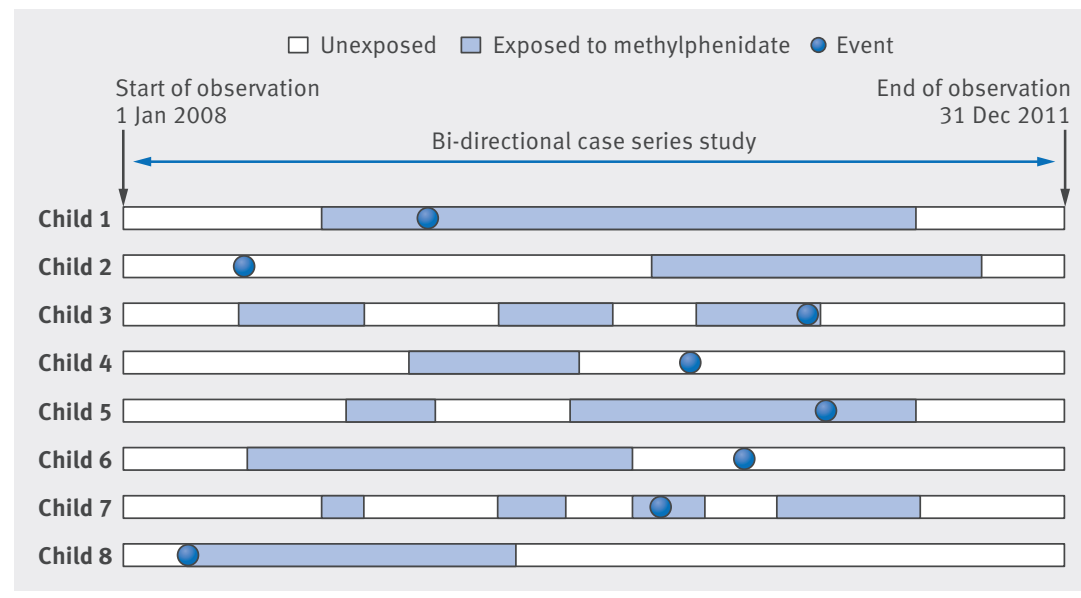

Fig 1 | Design of self controlled case series study in children and young people with ADHD and cardiovascular adverse events control. This method therefore controls for known and unknown confounders specific to the individual patient that do not vary over time, such as sex and genetic factors. The method enables comparisons in a population of individuals who have both the adverse event and exposure of interest. ${ }^{1617}$ This design can be particularly useful when secondary healthcare data are used for pharmacoepidemiological research when limited information is available on potential confounders. ${ }^{18}$ Figure 1 shows how we planned our design for an individual with respect to exposure applying a bi-directional self controlled case-series design.

\section{Patient involvement}

No patients were involved in setting the research question or the outcome measures, nor were they involved in developing plans for design or implementation of the study. No patients were asked to advise on interpretation or writing up of results. There are no plans to disseminate the results of the research to study participants or the relevant patient community.

\section{Participants}

All data used were obtained from secondary electronic records for the study participants. These were all patients aged $\leq 17$ who had at least one recorded diagnosis of ADHD (ICD-10 code F90), had started taking methylphenidate (ATC (Anatomical Therapeutic Chemical) code N06BA04), and had an incident cardiovascular adverse event with a recorded diagnosis during the study period (1 January 2008 and 31 December 2011). Patients were considered as new users of methylphenidate if they had not received a prescription during the preceding year.

Cardiovascular adverse events were defined as a recorded diagnosis (primary or secondary) for arrhythmias (codes I44, I45, I47, I48, I49), hypertension (code I10-I15), myocardial infarction (code I21), ischemic stroke (code I63), or heart failure (code I50). For patients with multiple diagnoses recorded, we included only the first event to avoid potential bias of the second event being influenced by the first event. ${ }^{16}$

The observation period started on 1 January 2008 and ended on 31 December 2011. There were five cases of death after the diagnosis of a cardiovascular adverse event, and for these cases we censored follow-up time at date of death.

Figure 2 shows a flow chart detailing the selection of participants from the national health insurance claims database for inclusion in this study.

\section{Assessment of exposure}

The index date was defined as the first date of prescription of methylphenidate. We calculated the length of exposure using information on prescription date and prescribed days in the database. Person time was divided into methylphenidate exposure risk periods: 1-3 days, 4-7 days, 8-14 days, 15-28 days, 29-56 days, and $>56$ days after prescription of the drug. Patients contributed to consecutive exposure risk periods while they were continuously exposed to the drug. All remaining time before and after exposure was considered as unexposed 


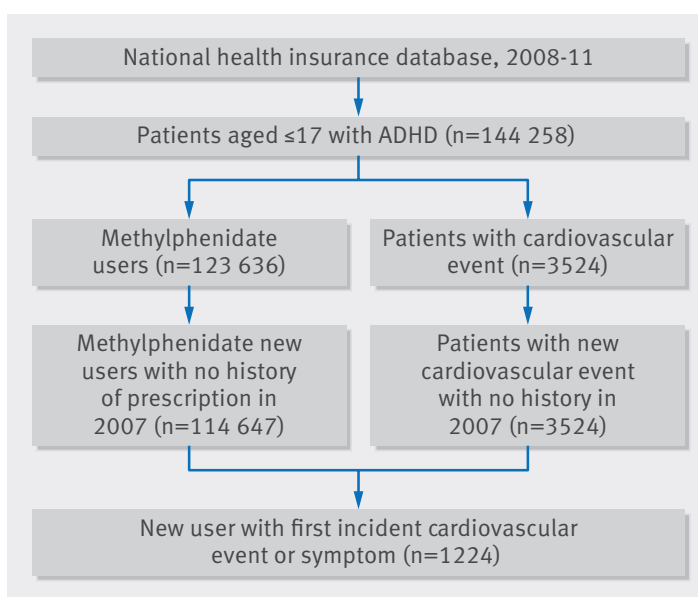

Fig 2 Selection of study participants from national health insurance database in self controlled case series design in children and young people with ADHD and cardiovascular adverse events

time. The exposure risk periods were defined based on the quantiles for the distribution of prescribed days.

\section{Time varying confounders}

We included the following time varying covariates in the model: age, comorbidities (depressive episode (codes F32F33, F34.1, F41.2), tic disorders (code F95), emotional disorders with onset specific to childhood (code F93), conduct disorders (code F91), manic episodes (code F30), bipolar affective disorders (code F31), and mental retardation (codes F70-79) and use of comedications (atomoxetine, antipsychotics (ATC code N05A), antidepressants (code N06A), anxiolotics (code N05B), antiepileptics (code N03A), and anticholinergic agents (code N04A)). Atomoxetine, a non-stimulant treatment for ADHD, is approved only for secondary treatment. We could not find any participants taking just atomoxetine-that is, all records of atomoxetine were prescriptions with methylphenidate.

\section{Statistical analysis}

We determined the duration of exposure in the study period and calculated incidence rates for cardiovascular events in each of the exposure risk periods. Incident rate ratios in periods of exposure to methylphenidate compared with unexposed periods were calculated with conditional Poisson regression. We estimated the adjusted incidence rate ratios and their $95 \%$ confidence intervals for exposure overall and for each predetermined exposure risk period. Incidence rate ratios were calculated for each outcome event separately.

Additional analysis was performed according to the presence of congenital heart disease (ICD-10 Q20-Q24), and we also conducted a subgroup analysis according to the daily prescribed dose of methylphenidate (low and high). The low dose group included patients prescribed less than $27 \mathrm{mg}$ a day of methylphenidate (median value), and the high dose group included patients with prescriptions of $27 \mathrm{mg}$ or more.

We conducted several sensitivity analyses in which we examined additional risk periods: a washout period, the day of initiation, and a pre-initiation exposure risk period. The washout period was used to account for the possibility that patients might not take their medicine strictly according to the prescription instructions and might be exposed to drugs for longer than prescribed. Washout periods were defined as 1-3 days, 4-7 days, and 8-14 days after the end date of prescription supply. The day of initiation was also examined separately, and we also included two consecutive 30 day pre-initiation exposure risk periods before the date the drug was first prescribed. This was done to account for the potential that the occurrence of the adverse event could influence exposure-that is, those patients who experience a cardiovascular event might be more or less likely to be started on methylphenidate.

The statistical application program SAS (release 9.4) was used for all statistical analyses. We considered a two tailed value of $\mathrm{P}<0.05$ to be significant.

\section{Results}

We identified and included 1224 eligible participants in the final analysis; 864 had arrhythmias, 396 had disease, 52 had myocardial infarction, 67 had ischaemic stroke, and 44 had heart failure. The mean duration of methylphenidate exposure was 0.5 years for all events except heart failure, which was 0.3 years. The median age at first exposure was 11-13 and median age at first event was $11-13$. Boys constituted $75-80 \%$ of each of the cohorts. The prevalence of comorbidities varied across adverse events. Depressive episode was the most common comorbidity ( $n=15,29 \%$ in participants with myocardial infarction), though only nine (13.4\%) participants with ischemic stroke had this condition. In contrast, mental retardation occurred in 12 (18\%) participants with an ischemic stroke, while only two (5\%) with heart failure had this condition. Antidepressants and antipsychotics were often coprescribed (15$27 \%)$. Antiepileptics were used in 17 (25\%) with ischemic stroke, though these drugs were used in only $68(8 \%)$ of patients with arrhythmia (table 1$)$.

There was an increased risk of arrhythmia for all risk periods combined (adjusted incidence rate ratio 1.61, 95\% confidence interval 1.48 to 1.74 ), with the highest risk observed on days one to three after the start of treatment (2.01, 1.74 to 2.31). The risk of arrhythmia reduced but remained significantly higher in the subsequent risk periods but was not significantly raised with longer term treatment ( $>56$ days) (table 2 ). While there was no increased risk of hypertension with methylphenidate exposure overall, when exposure time was divided into several risk periods after initiation, a higher risk was observed on days four to seven after the start of treatment. For myocardial infarction, no increased risk was observed overall, though risk was significantly raised for exposure up to 56 days only. No increased risk was observed with methylphenidate exposure for ischaemic stroke or heart failure. Incidence rates and age adjusted rate ratios are presented in the appendix.

Subgroup analysis for arrhythmia showed that risk was higher in patients with than without congenital heart disease (incidence rate ratio 3.49 (95\% confidence interval 2.33 to 5.22) $v 1.34$ (1.23 to 1.46); table 3). 


\begin{tabular}{|c|c|c|c|c|c|}
\hline & Arrhythmias & Hypertension & Myocardial infarction & Ischemic stroke & Heart failure \\
\hline No of patients & 864 & 396 & 52 & 67 & 44 \\
\hline Median (IQR) duration of exposure (years) & $0.5(0.1-1.3)$ & $0.5(0.1-1.3)$ & $0.5(0.1-1.1)$ & $0.5(0.2-1.3)$ & $0.3(0.1-0.8)$ \\
\hline Median (IQR) duration of exposure before events (years) & $0.04(0-0.48)$ & $0.04(0-0.48)$ & $0(0-0.26)$ & $0(0-0.42)$ & $0(0-0.13)$ \\
\hline Median (IQR) duration of exposure after events (years) & $0.20(0.02-0.75)$ & $0.17(0-0.70)$ & $0.17(0.04-0.91)$ & $0.27(0.04-0.83)$ & $0.12(0-0.50)$ \\
\hline Median (IQR) age at first exposure & $13(10-15)$ & $13(11-15)$ & $11(8-14)$ & $11(8-14)$ & $12(10-16)$ \\
\hline Median (IQR) age at first outcome & $13(10-15)$ & $13(10-16)$ & $11(8-14)$ & $11(8-14)$ & $13(9-15)$ \\
\hline No (\%) of boys & $674(78)$ & $317(80)$ & $39(75)$ & $51(76)$ & $33(75)$ \\
\hline No (\%) with congenital heart disease & $46(5.3)$ & $31(7.8)$ & $3(5.8)$ & $2(3.0)$ & $7(15.9)$ \\
\hline \multicolumn{6}{|l|}{ No (\%) with comorbidity* (ICD-10 code): } \\
\hline Depressive episode (F32-F33, F34.1, F41.2) & $223(25.8)$ & $101(25.5)$ & $15(28.9)$ & $9(13.4)$ & $12(27.3)$ \\
\hline Tic disorders (F95) & $76(8.8)$ & $35(8.8)$ & $7(13.5)$ & $6(9.0)$ & $2(4.6)$ \\
\hline Emotional disorders with onset specific to childhood (F93) & $77(8.9)$ & $31(7.8)$ & $5(9.6)$ & $8(11.9)$ & $5(11.4)$ \\
\hline Conduct disorders (F91) & $82(9.5)$ & $34(8.6)$ & $1(1.9)$ & $3(4.5)$ & $3(6.8)$ \\
\hline Manic episode (F30) & $7(0.8)$ & $2(0.5)$ & $0(0)$ & $0(0)$ & $0(0)$ \\
\hline Bipolar affective disorder (F31) & $44(5.1)$ & $15(3.8)$ & $2(3.9)$ & $3(4.5)$ & $1(2.3)$ \\
\hline Mental retardation (F70-F79) & $75(8.7)$ & $30(7.7)$ & $4(7.7)$ & $12(17.9)$ & $2(4.6)$ \\
\hline \multicolumn{6}{|l|}{ No (\%) with comedicationt (ATC code): } \\
\hline Atomoxetine & $15(1.7)$ & $5(1.2)$ & $2(2.9)$ & $2(2.9)$ & $0(0.0)$ \\
\hline Antipsychotics (ATC: N05A) & $114(13.2)$ & $55(13.9)$ & $7(13.5)$ & $5(7.5)$ & $2(4.6)$ \\
\hline Antidepressants (ATC: N06A) & $181(21.0)$ & $78(19.7)$ & $8(15.4)$ & $12(17.9)$ & $12(27.3)$ \\
\hline Antiepileptics (ATC: N03A) & $68(7.9)$ & $37(9.3)$ & $5(9.6)$ & $17(25.4)$ & $7(15.9)$ \\
\hline Anticholinergic agents (ATC: N04A) & $31(3.6)$ & $19(4.8)$ & $3(5.8)$ & $2(3.0)$ & $0(0)$ \\
\hline Anxiolotics (ATC: N05B) & $71(8.2)$ & $36(9.1)$ & $0(0)$ & $4(6.0)$ & $4(9.1)$ \\
\hline
\end{tabular}

IQR=interquartile range.

*Defined as at least one diagnosis or prescription between 1 January 2008 and 31 December 2011.

tDefined as coprescription with methylphenidate during same period.

Table 2 | Risk of cardiovascular adverse events before and after treatment with methylphenidate

\begin{tabular}{|c|c|c|c|c|c|c|c|c|c|c|}
\hline \multirow{3}{*}{$\begin{array}{l}\text { Risk period } \\
\text { Primary analysi }\end{array}$} & \multicolumn{2}{|c|}{ Arrhythmias } & \multicolumn{2}{|c|}{ Hypertension } & \multicolumn{2}{|c|}{ Myocardial infarction } & \multicolumn{2}{|c|}{ Ischemic stroke } & \multicolumn{2}{|c|}{ Heart failure } \\
\hline & $\begin{array}{l}\text { No of } \\
\text { patients }\end{array}$ & $\mathrm{RR}(95 \% \mathrm{Cl}) *$ & $\begin{array}{l}\text { No of } \\
\text { patients }\end{array}$ & $\operatorname{RR}(95 \% \mathrm{Cl})^{*}$ & $\begin{array}{l}\text { No of } \\
\text { patients }\end{array}$ & $\operatorname{RR}(95 \% \mathrm{Cl})^{*}$ & $\begin{array}{l}\text { No of } \\
\text { patients }\end{array}$ & $\operatorname{RR}(95 \% \mathrm{Cl})^{*}$ & $\begin{array}{l}\text { No of } \\
\text { patients }\end{array}$ & $\operatorname{RR}(95 \% \mathrm{Cl})^{*}$ \\
\hline & \multicolumn{10}{|c|}{ Primary analysis } \\
\hline Exposed & 234 & 1.61 (1.48 to 1.74$)$ & 92 & 1.07 (0.94 to 1.22$)$ & 10 & 1.33 (0.90 to 1.98$)$ & 10 & 0.70 (0.49 to 1.01$)$ & 4 & $0.54(0.30$ to 0.96$)$ \\
\hline \multicolumn{11}{|c|}{ Time since initiation of methylphenidate (days) } \\
\hline $1-3$ & 24 & 2.01 (1.74 to 2.31$)$ & 6 & 1.01 (0.77 to 1.31$)$ & 0 & NA & 1 & 1.16 (0.61 to 2.22 ) & 0 & NA \\
\hline $15-28$ & 45 & $1.29(1.16$ to 1.44$)$ & 19 & 1.03 (0.88 to 1.22$)$ & 2 & 1.95 (1.17 to 3.23$)$ & 0 & NA & 1 & 0.94 (0.44 to 2.00$)$ \\
\hline $29-56$ & 51 & $1.54(1.38$ to 1.71$)$ & 18 & $0.90(0.75$ to 1.08$)$ & 3 & 2.57 (1.65 to 4.02$)$ & 4 & $1.00(0.68$ to 1.47$)$ & 0 & NA \\
\hline$>56$ & 53 & $1.03(0.91$ to 1.15$)$ & 30 & $1.14(0.97$ to 1.35$)$ & 2 & 0.67 (0.33 to 1.37$)$ & 2 & $0.38(0.22$ to 0.65$)$ & 2 & $0.17(0.07$ to 0.43$)$ \\
\hline
\end{tabular}

$\mathrm{NA}=$ not applicable.

* Rate ratio adjusted for age, comorbidity, and co-medication in time varying method.

\begin{tabular}{|c|c|c|c|c|c|c|c|c|c|c|}
\hline \multirow[b]{2}{*}{ Risk period } & \multicolumn{2}{|c|}{ Arrhythmias } & \multicolumn{2}{|c|}{ Hypertension } & \multicolumn{2}{|c|}{ Myocardial infarction } & \multicolumn{2}{|c|}{ Ischemic stroke } & \multicolumn{2}{|c|}{ Heart failure } \\
\hline & $\begin{array}{l}\text { No of } \\
\text { patients }\end{array}$ & $\operatorname{RR}(95 \% \mathrm{Cl})^{*}$ & $\begin{array}{l}\text { No of } \\
\text { patients }\end{array}$ & $\operatorname{RR}(95 \% \mathrm{Cl})^{*}$ & $\begin{array}{l}\text { No of } \\
\text { patients }\end{array}$ & $\mathrm{RR}(95 \% \mathrm{Cl})^{*}$ & $\begin{array}{l}\text { No of } \\
\text { patients }\end{array}$ & $\operatorname{RR}(95 \% \mathrm{Cl})^{*}$ & $\begin{array}{l}\text { No of } \\
\text { patients }\end{array}$ & $\operatorname{RR}(95 \% \mathrm{Cl})^{*}$ \\
\hline \multicolumn{11}{|c|}{ Congenital heart disease } \\
\hline Yes & 46 & 3.49 (2.33 to 5.22$)$ & 31 & 0.95 (0.58 to 1.56$)$ & 3 & NA & 2 & NA & 7 & NA \\
\hline No & 818 & $1.34(1.23$ to 1.46$)$ & 365 & 0.99 (0.87 to 1.13$)$ & 49 & 1.36 (0.89 to 2.07 & 65 & $0.61(0.42$ to 0.89$)$ & 37 & $0.38(0.20$ to 0.72$)$ \\
\hline
\end{tabular}

\begin{tabular}{|c|c|c|c|c|c|c|c|c|c|c|}
\hline Low $(<27)$ & 814 & 1.42 (1.29 to 1.56$)$ & 375 & 1.03 (0.88 to 1.20$)$ & 51 & 1.49 (0.97 to 2.30$)$ & 66 & 0.93 (0.62 to 1.38$)$ & 40 & 0.18 (0.07 to 0.46$)$ \\
\hline High $(\geq 27)$ & 622 & $1.42(1.29$ to 1.55$)$ & 285 & 1.04 (0.91 to 1.19$)$ & 30 & 1.39 (0.88 to 2.20$)$ & 34 & 0.27 (0.15 to 0.49$)$ & 26 & 1.06 (0.56 to 2.02$)$ \\
\hline
\end{tabular}

$\mathrm{NA}=$ not applicable.

* Rate ratio adjusted for age, comorbidity, and co-medication in time varying method.

tCase series analysis according to daily dose conducted in dataset of patients with prescriptions $<27 \mathrm{mg}$ of daily dose, and those with prescriptions $\geq 27 \mathrm{mg}$. Patient can be duplicated in both

dataset as they might change their dose in study period. 


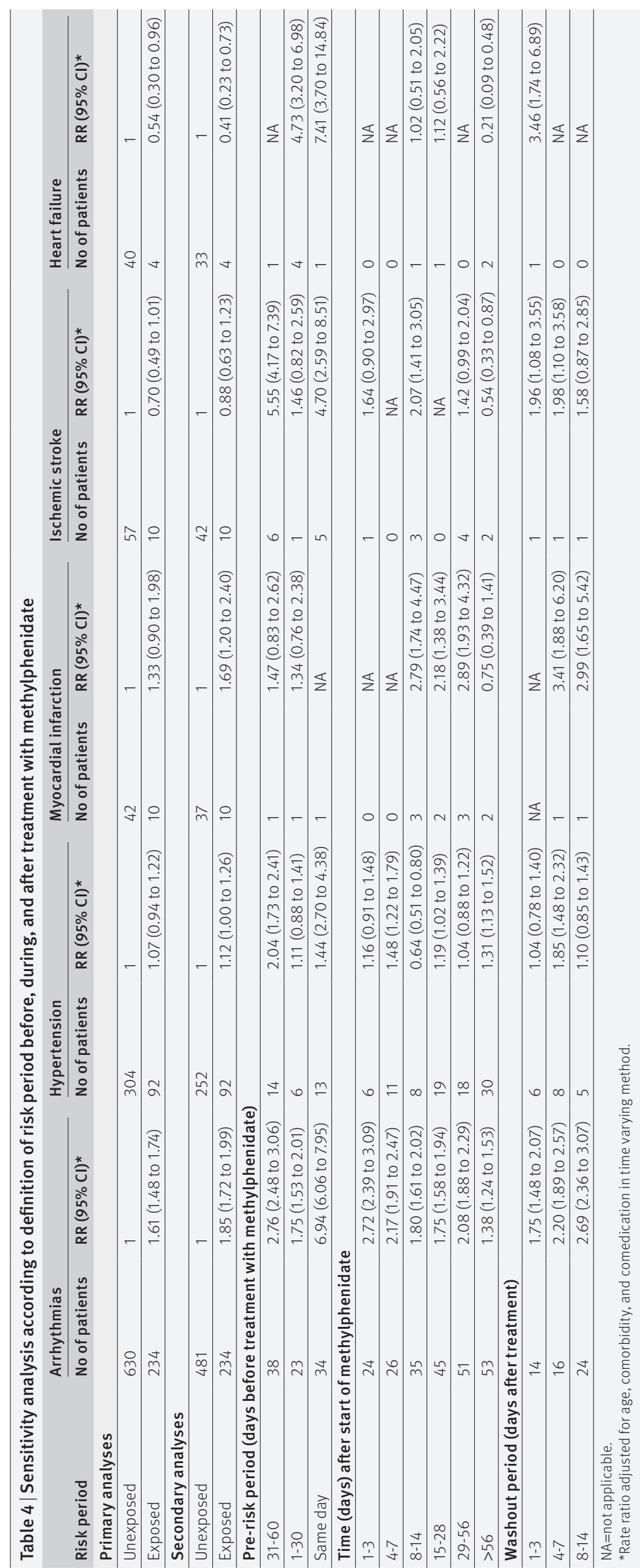

There was no differential effect for hypertensive disease in children with or without congenital heart disease. Numbers of children with congenital heart disease were low for the other outcomes. No differential effects between low and high daily doses of methylphenidate were observed for any outcome.

Sensitivity analysis showed higher risk estimates in the exposed period than obtained in the primary analysis (table 4). The sensitivity analysis for the overall risk of myocardial infarction in exposed time was significant (incidence rate ratio 1.69, 95\% confidence interval 1.20 to 2.40 ), while in the primary analysis the effect was not significant (1.33, 0.90 to 1.98). No increased risk of myocardial infarct was found in the risk period before exposure, while in the washout period the risk was three times higher than in unexposed time.

\section{Discussion}

Principal findings

This study found a significantly increased overall risk of arrhythmia associated with treatment with methylphenidate in children and young people with ADHD. We also identified associations according to the timing of exposure for other cardiovascular adverse events. While the risk of myocardial infarction was not significant overall, we found an increased risk after the first week of treatment, which remained significantly raised for the first two months of continuous treatment. Though there was an increased risk for arrhythmia overall, the risk was substantially higher in patients with existing congenital heart disease. These results are consistent with the biological plausibility that the mechanism of action relates to the effect of methylphenidate on the heart rate. ${ }^{19} 20$ Delayed effects would be expected with myocardial infarction, while more immediate effects would be expected with arrhythmias, as we observed.

\section{Comparison with other studies}

Previous research has found no evidence of increased risk of stroke or myocardial infarction with methylphenidate, which was consistent with our overall risk. Our time based risk analysis, however, suggests that the risk of myocardial infarction might be higher in the early risk periods (8-14, 15-28, and 29-56 days). This result is of concern and warrants further research. The lack of increased risk for all events with long term treatment might reflect discontinuation of treatment in those who experienced a cardiovascular adverse event or it might be influenced by the limited long term continuous use of methylphenidate in this population.

We found no previous observational studies investigating the risk of heart failure with methylphenidate. There was no increased risk of heart failure in our study, though numbers of events were low. The risk of arrhythmia was increased for all risk periods up to 56 days, and, in particular, risk was highest in patients with congenital heart disease. While the risk of arrhythmia was lower in those without congenital heart disease, a significant risk was still present. The risk of hypertension varied across risk periods and was 
not consistent across the primary and sensitivity analyses. Some form of detection bias could have influenced the results for hypertension as it is a condition that is non-symptomatic and might have been detected only through routine tests at scheduled follow-up appointments with healthcare providers. We did find a significantly increased risk of hypertension in the four to seven day risk period, which would correspond to the first follow-up visit after the start of treatment. In contrast, all the other cardiovascular events assessed were symptomatic events, the symptoms of which would precipitate attendance independent of a planned visit, hence a more accurate assessment of timing of the event.

We used one of the largest population datasets, and the study design was robust to confounding. A systematic review of previous observational research investigating the risk of cardiovascular adverse events with methylphenidate reported that six out of seven studies in children and adolescents did not show an association. Because of the low number of events, however, many of these previous studies did not have sufficient statistical power, and the study designs used meant that confounding could not be ruled out. ${ }^{8}$

\section{Methodological considerations}

To the best of our knowledge, this is the first population based self controlled case series study focusing on the risk of a comprehensive list of cardiovascular adverse events in children exposed to methylphenidate. We also assessed the temporal association between the start of treatment with methylphenidate and cardiovascular adverse events. Previous research has shown that the self controlled case series method produces similar risk estimates to a new users cohort study design and has the advantage of controlling for constant patient specific confounders because of its within person study design. ${ }^{21}$ Previous studies with cohort designs examined whether children who take methylphenidate have a higher risk of cardiovascular events compared with those who do not. Our study, however, determines whether methylphenidate use "triggers" the occurrence of a cardiovascular adverse event within a patient. Our population included only those children who had the exposure and the event during the study period. As described by Maclure and colleagues, the analysis of a within person study design can be summarized by the expression "why now" rather than "why me" as in a cohort or case-control study. ${ }^{22}$

We conducted several sensitivity analyses to verify the robustness of our results by applying a washout period, day of initiation, and a pre-initiation exposure risk period. The pre-initiation exposure risk period was used to test the assumption of the self controlled case series design that the occurrence of an event must not alter the probability of subsequent exposures. ${ }^{1617}$ We found that the incidence risk ratios were high in the periods before exposure, suggesting that the probability of exposure to methylphenidate is increased after a cardiovascular adverse event for all events except myocardial infarction. By categorizing this period separately from the reference (unexposed) period, we found that the incidence rate ratios in the periods after exposure in the sensitivity analyses were higher than those in our primary analysis (table 4 ). This is biologically plausible because ADHD itself might be highly correlated with cardiovascular disease. Several observational studies have reported that patients with ADHD have more pre-existing cardiovascular disease than the general population. ${ }^{2324}$ Several previous studies applying self controlled case series designs have defined the periods before exposure to control for potential bias. ${ }^{25-27}$ Our sensitivity analysis suggests that our main analysis might be biased toward the null and might have underestimated the effects of methylphenidate.

Another assumption of the self controlled case series design is that recurrent adverse events must be independent-that is, the occurrence of one event must not alter the probability of a subsequent event occurring. ${ }^{16} 17$ In our study, once a person has had a cardiovascular adverse event, their short term risk of experiencing another might be increased. Therefore, events might cluster within episodes. We found $10 \%$ of children and adolescents had a recurrent diagnosis of cardiovascular adverse events within 30 days. We overcame this limitation, however, by including only the first event.

The final assumption of the self controlled case series design is that the occurrence of the event of interest must not censor or affect the length of the observation period. ${ }^{161721}$ Our study population covered children and adolescents, and there were only five deaths after the diagnosis of cardiovascular adverse events in 1224 participants. Farrington and colleagues ${ }^{28}$ and previous studies using a self controlled case series design ${ }^{2126}$ have shown that this method might be robust to failure of this assumption. Additionally we found that the main results did not change when we limited analysis to children who survived until the end of the study (data not shown).

\section{Strengths and limitations}

This study had several strengths. Our study included the entire Korean population and used the claims database for all patients with ADHD collected by the national health insurance program. Until recently, evidence of the cardiovascular effects of drugs for ADHD was limited to a few observational studies. As cardiovascular adverse events are rare in adolescence, these studies had insufficient power to detect rare events. Our results representing a large population in a real world setting suggest that particular cardiovascular adverse events could be associated with exposure, consistent with the mechanism of action of methylphenidate on heart rate. While we did find an increased relative risk, the absolute risk is likely to be low.

Secondly, we performed a self controlled case series study, which controls for patient specific measured and unmeasured confounders that do not vary over time and has previously been shown to produce similar risk estimates to a new user cohort study design. ${ }^{21}$ While the self controlled case series design adjusts 
implicitly for constant patient specific confounders, it is possible that the association observed might be biased because of time varying confounders. Antidepressants, antipsychotics, or antiepileptics are often coprescribed with methylphenidate and could explain some of the association found with cardiovascular adverse events. ${ }^{29} 30$ Though we adjusted all our analyses for time varying comorbidities and comedications, it is possible that other unmeasured time varying confounders could have influenced our results. For example, there might be differences in severity of ADHD symptoms, substance use, and precipitating factors that could have influenced both the occurrence of a cardiovascular adverse event and methylphenidate exposure.

The increased risk in the period before treatment suggests that children with cardiovascular events, except myocardial infarction, were started on methylphenidate treatment. This might reflect prescribing in contraindicated patients and is in itself of concern and might indicate that prescribers consider only myocardial infarction to be a contraindication to methylphenidate use. Additionally we found an increased risk of cardiovascular outcomes in the washout period. There are several possible explanations for this. Firstly, the exposure duration might have been underestimated-that is, patients might have still been taking the drug for longer than expected because of non-compliance. Secondly, methylphenidate comes in slow release preparations and is metabolized by the liver, either of which could have implications for people who are slow metabolizers, in whom there might be some potential for drug activity beyond the expected supply.

Our findings should be interpreted with caution. Firstly, there is the potential for inaccuracy of coding and incompleteness of records. The outcome measures were limited to patients with diagnoses of cardiovascular adverse events, and we could have missed outcomes not diagnosed. A validation study comparing the diagnosis derived from the health insurance database with the actual diagnosis recorded in patients' medical records in Korea found that the overall positive predictive value of diagnoses was about $70 \% .{ }^{13}$ There is no reason to suspect a differential rate of misclassification in exposed and unexposed periods so this is unlikely to introduce bias in our study. Secondly, we defined the study outcome as a primary or secondary diagnosis for cardiovascular adverse events. Though this is likely to have increased the power of our study by capturing all possible cases of cardiovascular events, some minor events might have been captured. Thirdly, given that this is an observational study, non-compliance to the drug prescribed could have influenced our results. If patients did not take their drug after prescription, our exposure measure will be misclassified, which would affect our results. However, there was a median of only one day between repeat prescriptions. This high persistence rate of methylphenidate meant non-compliance was unlikely in our participants. Finally, a safety warning for methylphenidate associated with cardiovascular adverse events was issued in Korea in December
2009. We have assumed that this warning will not have influenced medical practice in our study (2007-11), though it possibly had some influence on the results.

\section{Conclusions}

Methylphenidate exposure in children/young people with diagnosis of ADHD is associated with arrhythmia and potentially with myocardial infarction in specific time periods of use. With the increased use of drugs for ADHD globally, the benefits of methylphenidate should be carefully weighed against the potential cardiovascular risks of these drugs in children and adolescents.

We acknowledge Emmae Ramsay, School of Pharmacy and Medical Sciences, University of South Australia, Adelaide SA 5001, Australia, who contributed to the development of the statistical analysis code used in this research.

Contributors: All authors contributed to study design and interpretation of data. J-YS and B-JP acquired the database. J-YS and NLP had the main responsibility for statistical analysis, but all authors contributed. J-YS, EER, and NLP wrote the manuscript, and all authors reviewed and commented on drafts and approved the final manuscript and the decision to submit for publication. J-YS is guarantor, accept full responsibility for the research, had access to the data and controlled the decision to publish.

Funding: This research received no specific grant from any funding agency in the public, commercial, or not-for-profit sectors. EER was supported by an NHMRC fellowship (GNT1110139). NP was supported by an NHMRC early career fellowship (GNT1035889).

Competing interests: All authors have completed the ICMJE uniform disclosure form at www.icmje.org/coi_disclosure.pdf and declare: no support from any organization for the submitted work; no financial relationships with any organizations that might have an interest in the submitted work in the previous three years; no other relationships or activities that could appear to have influenced the submitted work.

Ethical approval: This study was approved by the institutional review board of the Korea Institute of Drug Safety and Risk Management, Seoul (study ID: IRB-2014-002). Obtaining informed consent from the study population was waived by the board.

Data sharing: No additional data available.

Transparency: The lead author affirms that the manuscript is an honest, accurate, and transparent account of the study being reported; that no important aspects of the study have been omitted; omitted; and that any discrepancies from the study as planned (and, if relevant, registered) have been explained.

This is an Open Access article distributed in accordance with the Creative Commons Attribution Non Commercial (CC BY-NC 3.0) license, which permits others to distribute, remix, adapt, build upon this work non-commercially, and license their derivative works on different terms, provided the original work is properly cited and the use is non-commercial. See: http://creativecommons.org/licenses/ by-nc/3.0/.

Wigal SB. Efficacy and safety limitations of attention-deficit hyperactivity disorder pharmacotherapy in children and adults. CNS Drugs 2009;23(Suppl 1):21-31. doi:10.2165/00023210-200923000-00004

2 Bange F, Le Heuzey MF, Acquaviva E, Delorme R, Mouren MC. [Cardiovascular risks and management during Attention Deficit Hyperactivity Disorder treatment with methylphenidate]. Arch Pediatr 2014;21:108-12. doi:10.1016/j.arcped.2013.11.001.

3 Gould MS, Walsh BT, Munfakh JL, et al. Sudden death and use of stimulant medications in youths. Am J Psychiatry 2009;166:992-1001. doi:10.1176/appi.ajp.2009.09040472.

4 Schelleman H, Bilker WB, Strom BL, et al. Cardiovascular events and death in children exposed and unexposed to ADHD agents. Pediatrics 2011:127:1102-10. doi:10.1542/peds.2010-3371.

5 Cooper WO, Habel LA, Sox CM, et al. ADHD drugs and serious cardiovascular events in children and young adults. N Engl J Med 2011:365:1896-904. doi:10.1056/NEJMoa1110212.

6 Winterstein AG, Gerhard T, Kubilis P, et al. Cardiovascular safety of central nervous system stimulants in children and adolescents: population based cohort study. BMJ 2012;345:e4627. doi:10.1136/ bmi.e4627.

Winterstein AG, Gerhard T, Shuster J, Johnson M, Zito JM, Saidi A. Cardiac safety of central nervous system stimulants in children and adolescents with attention-deficit/hyperactivity disorder. Pediatrics 2007;120:e1494-501. doi:10.1542/peds.2007-0675. 
8 Westover AN, Halm EA. Do prescription stimulants increase the risk of adverse cardiovascular events?: A systematic review. BMC CardiovasC Disord 2012:12:41. doi:10.1186/1471-2261-12-41.

9 Munk K, Gormsen L, Kim WY, Andersen NH. Cardiac Arrest following a Myocardial Infarction in a Child Treated with Methylphenidate. Case Rep Pediatr 2015;2015:905097. doi:10.1155/2015/905097.

10 Wikström G, Kvidal P, Hagström E. [Life-threatening heart failure caused by ADHD medication. Five case reports described]. Lakartidningen 2012;109:2016-8.

11 Martinez-Raga J, Knecht C, Szerman N, Martinez MI. Risk of serious cardiovascular problems with medications for attention-deficit hyperactivity disorder. CNS Drugs 2013;27:15-30. doi:10.1007/s40263-012-0019-9.

12 Shin IY, Lee SH, Shin SM, Shin HN, Park BJ. Regulatory action and moderate decrease in methylphenidate use among ADHD diagnosed patients aged five and under in Korea. Regul Toxicol Pharmacol 2015;72:244-8. doi:10.1016/j.yrtph.2015.04.022.

13 Park BJ, Sung JH, Park KD, Seo SW, Kim SW. Report of the evaluation for validity of discharged diagnoses in Korean health insurance database.Seoul National University, 2003:19-52.

14 Park JK, Kim KS, Kim CB, et al. The accuracy of ICD codes for cerebrovascular diseases in medical insurance claims. J Prev Med Public Health 2000;33:76-82. http://www.koreamed.org/SearchBasic. php?RID=2056KJPM/2000.33.1.76\&DT=1

15 Kimm H, Yun JE, Lee S-H, Jang Y, Jee SH. Validity of the diagnosis of acute myocardial infarction in korean national medical health insurance claims data: the Korean heart study (1). Korean Circ J 2012;42:10-5. doi:10.4070/kcj.2012.42.1.10

16 Whitaker HJ, Farrington CP, Spiessens B, Musonda P. Tutorial in biostatistics: the self-controlled case series method. Stat Med 2006;25:1768-97. doi:10.1002/sim.2302.

17 Whitaker HJ, Hocine MN, Farrington CP. The methodology of self-controlled case series studies. Stat Methods Med Res 2009;18:7-26. doi:10.1177/0962280208092342.

18 Hallas J, Pottegård A. Use of self-controlled designs in pharmacoepidemiology. J Intern Med 2014;275:581-9. doi:10.1111/joim.12186.

19 Samuels JA, Franco K Wan F Sorof IM. Effect of stimulants on 24-h ambulatory blood pressure in children with ADHD: a double-blind, randomized, cross-over trial. Pediatr Nephrol 2006;21:92-5. doi:10.1007/s00467-005-2051-1.

20 Stowe CD, Gardner SF, Gist CC, Schulz EG, Wells TG. 24-hour ambulatory blood pressure monitoring in male children receiving stimulant therapy. Ann Pharmacother 2002;36:1142-9. doi:10.1345/aph.1A367.

21 Ramsay EN, Pratt NL, Ryan P, Roughead EE. Proton pump inhibitors and the risk of pneumonia: a comparison of cohort and self-controlled case series designs. BMC Med Res Methodol 2013;13:82. doi:10.1186/1471-2288-13-82
22 Maclure M. 'Why me?' versus 'why now?'-differences between operational hypotheses in case-control versus case-crossove studies. Pharmacoepidemiol Drug Saf 2007:16:850-3. doi:10.1002/ pds.1438.

23 Vetter VL, Elia J, Erickson C, et al. American Heart Association Council on Cardiovascular Disease in the Young Congenital Cardiac Defects Committee American Heart Association Council on Cardiovascular Nursing. Cardiovascular monitoring of children and adolescents with heart disease receiving medications for attention deficit/hyperactivity disorder [corrected]: a scientific statement from the American Heart Association Council on Cardiovascular Disease in the Young Congenital Cardiac Defects Committee and the Council on Cardiovascular Nursing. Circulation 2008;117:2407-23. doi:10.1161/ CIRCULATIONAHA.107.189473.

24 Kraut AA, Langner I, Lindemann C, et al. Comorbidities in ADHD children treated with methylphenidate: a database study. BMC Psychiatry 2013;13:11. doi:10.1186/1471-244X-13-11.

25 Douglas IJ, Langham J, Bhaskaran K, Brauer R, Smeeth L. Orlistat and the risk of acute liver injury: self controlled case series study in UK Clinical Practice Research Datalink. BM/ 2013:346:f1936. doi:10.1136/bmj.f1936.

26 Pratt NL, Ramsay EN, Kemp A, et al. Ranibizumab and risk of hospitalisation for ischaemic stroke and myocardial infarction in patients with age-related macular degeneration: a self-controlled case-series analysis. Drug Saf 2014;37:1021-7. doi:10.1007/ s40264-014-0231-2.

27 Maclure M, Fireman B, Nelson JC, et al. When should case-only designs be used for safety monitoring of medical products? Pharmacoepidemiol Drug Saf 2012;21(Suppl 1):50-61. doi:10.1002/ pds.2330.

28 Farrington CP, Whitaker HJ. Semiparametric analysis of case series data. J R Stat Soc Ser CAppl Stat 2006;55:553-94. doi:10.1111/j.1467-9876.2006.00554.X.

29 Swenson IR, Doucette S, Fergusson D. Adverse cardiovascular events in antidepressant trials involving high-risk patients: a systematic review of randomized trials. Can J Psychiatry 2006;51:923-9.

30 Murray-Thomas T, Jones ME, Patel D, et al. Risk of mortality (including sudden cardiac death) and major cardiovascular events in atypical and typical antipsychotic users: a study with the general practice research database. Cardiovasc Psychiatry Neurol 2013;2013:247486. doi:10.1155/2013/247486

(C) BMJ Publishing Group Ltd 2016

Appendix: Additional tabulated data 